\title{
A Rare Case of Mollaret's Meningitis Complicated by Chronic Intractable Migraine and Papilledema: Case Report and Review of Literature
}

\author{
Kinjal P. Gadhiya ${ }^{1}$, Vinod Nookala ${ }^{1}$ \\ 1. Internal Medicine, University of Pittsburgh Medical Center (UPMC) Pinnacle, Harrisburg, USA
}

Corresponding author: Kinjal P. Gadhiya, kinjal268@gmail.com

\begin{abstract}
Mollaret's meningitis is characterized by recurrent episodes of aseptic meningitis that last two to seven days and resolve spontaneously without any residual neurological deficit or complication. Viruses are the most common cause of aseptic meningitis and herpes simplex virus (HSV) type 2 has been noted as the most commonly associated virus in Mollaret's meningitis. We describe a rare case of a female who had four episodes of meningitis in a five-year period associated with chronic intractable migraine and papilledema attributed to Mollaret's meningitis.
\end{abstract}

Categories: Internal Medicine, Neurology, Infectious Disease

Keywords: mollaret, meningitis, migraine

\section{Introduction}

In 1944, French Neurologist Pierre Mollaret identified a rare form of recurrent, benign meningitis, which was named "Mollaret's meningitis." It is characterized by episodes of aseptic meningitis that resolve spontaneously with no residual neurological deficit [1]. The rate of recurrence is variable, with episodes ranging in frequency from every few weeks to intervals of many years. The overall course of the disease has been documented to range from three to 28 years [1]. Herpes simplex virus type 2 (HSV-2) is the most commonly isolated pathogen in this condition. The acute phase may produce transient neurological symptoms but no permanent impairment. We describe the case of a female who presented with five episodes of meningitis in a five-year period associated with chronic intractable migraine and papilledema.

Received 01/30/2020 Review began 01/31/2020 Review ended 02/05/2020 Published 02/18/2020

() Copyright 2020 Gadhiya et al. This is an open access article distributed under the terms of the Creative Commons Attribution License CC-BY 4.0., which permits unrestricted use, distribution, and reproduction in any medium, provided the original author and source are credited.

\section{Case Presentation}

A 44-year-old female was admitted to the hospital with new-onset headache for one day. She had a very severe, dull, constant frontal headache, which was associated with photophobia, nausea, neck pain with movement, fever, and chills. She had no history of migraine. She had had a spinal steroid injection for back pain a few days prior to presentation. Her past medical history was significant for hypertension, hyperlipidemia, diabetes mellitus type 2 , non-occlusive coronary artery disease, hypothyroidism following thyroidectomy, bipolar disorder, chronic back pain due to lumbar disc protrusion, and bilateral upper extremity paresthesia associated with cervical spinal stenosis. Her family history was significant for heart disease, diabetes, hypertension, and seizure disorder, and her personal history included smoking. Physical exam revealed a fever of $102.0^{\circ} \mathrm{F}$ and positive Kernig's and Brudzinski signs. She remained alert and oriented. She had leukocytosis of 22700/microL, elevated hemoglobin of $16.6 \mathrm{~g} / \mathrm{dl}$, and elevated platelet of 467000/microL. Cerebrospinal fluid (CSF) polymerase chain reaction (PCR) was positive for HSV-2 while the CSF culture was negative (Table 1). She was treated with a 14-day course of intravenous (IV) acyclovir. The repeat CSF analysis in a month showed that the HSV-2 PCR was negative. 


\begin{tabular}{|c|c|c|c|c|c|c|}
\hline & $\begin{array}{l}\text { First } \\
\text { episode }\end{array}$ & $\begin{array}{l}\text { Follow-up in a } \\
\text { month }\end{array}$ & $\begin{array}{l}\text { Second } \\
\text { episode }\end{array}$ & $\begin{array}{l}\text { Admission for } \\
\text { (migraine) }\end{array}$ & $\begin{array}{l}\text { Third } \\
\text { episode }\end{array}$ & $\begin{array}{l}\text { Fourth } \\
\text { episode }\end{array}$ \\
\hline HSV-1 PCR & Negative & Negative & Negative & Negative & Negative & Negative \\
\hline HSV-2 PCR & Positive & Negative & Positive & Negative & Positive & Negative \\
\hline $\begin{array}{l}\text { Nucleated cells }(\mathrm{N}=0-5 \\
\text { /CUMM) }\end{array}$ & 215 & 33 & 1268 & 0 & 230 & 3 \\
\hline RBC (N=0/CUMM) & 6 & 8 & 6 & 1 & 20 & 11 \\
\hline Neutrophils ( $\mathrm{N}=0-6 \%)$ & 7 & 0 & 18 & $\mathrm{~N} / \mathrm{A}$ & 4 & $\mathrm{~N} / \mathrm{A}$ \\
\hline Lymphocytes ( $\mathrm{N}=40-80 \%)$ & 53 & 94 & 75 & N/A & 84 & N/A \\
\hline Monocytes ( $\mathrm{N}=15-45 \%)$ & 39 & 6 & 7 & N/A & 12 & N/A \\
\hline $\begin{array}{l}\text { Total protein (N=15-45 } \\
\mathrm{mg} / \mathrm{dL})\end{array}$ & 161 & 43 & 262 & 29 & 73 & 32 \\
\hline Glucose $(\mathrm{N}=40-70 \mathrm{mg} / \mathrm{dL})$ & 62 & 77 & 76 & 75 & 75 & 102 \\
\hline
\end{tabular}

\section{TABLE 1: CSF analysis during each episode of aseptic meningitis}

After the first episode of HSV-2 meningitis, she developed chronic, uncontrolled migraines with staring spells as well as myoclonic jerks related to migraine. Her migraine and myoclonic jerks were managed by valproic acid, gabapentin, sumatriptan, and oxycodone as needed, and botulinum toxin (Botox) injections every six weeks. She had a mild improvement of migraine and myoclonic jerks with this treatment over the next three years. During this time period, however, she was diagnosed with depression and post-traumatic stress disorder (PTSD) and treated with sertraline and venlafaxine.

Three years after the first episode, she had meningitis again, with a similar clinical presentation as before, with fever, headache, and positive Kernig’s and Brudzinski signs. The CSF study showed elevated opening pressure (OP) of $37 \mathrm{cmH} 2 \mathrm{O}$ and positive HSV-2 PCR (Table 1). She underwent treatment with IV acyclovir for 10 days, followed by oral valacyclovir for two weeks as prophylaxis. Electroencephalography (EEG) did not show any epileptiform activities. Head imaging was negative for any abnormalities. After the second episode of HSV-2 meningitis, her migraine symptoms had worsened in intensity, frequency, and duration, and she developed dizziness, vertigo, and transient hearing loss. Despite being on higher doses of gabapentin, valproic acid, sertraline, and venlafaxine, her migraine was not controlled and she resumed Botox injections, which were effective.

A year later, she was admitted for a severe intractable migraine attack with negative CSF, HSV, PCR, and CSF leukocytosis (Table 1). She did not have fever or neck stiffness. Upon discharge, she was started on topiramate for migraine prophylaxis. She subsequently developed an intermittent pulsatile headache with ptosis, blurred/double vision, and tinnitus. There was a concern for pseudotumor cerebri. Repeat lumbar puncture (LP) was done as an outpatient and showed normal OP - $13 \mathrm{~cm}$ of water and normal closing pressure (CP) - less than $10 \mathrm{~cm}$ of water. She had a dilated fundus exam, which showed papilledema without vision disturbances. Weight loss along with Botox injections somewhat helped with her headaches.

Eight months later, she had the third occurrence of aseptic meningitis with similar presentation as the previous episodes with fever, headache, and positive Kernig's and Brudzinski signs. LP showed OP - $22 \mathrm{~cm}$ of water and CP - $13 \mathrm{~cm}$ of water. CSF, HSV-2, and PCR were positive (Table 1) and she received treatment with IV acyclovir for two weeks. Her headache slightly improved upon completion of acyclovir while she continued other medications as previously described.

Again, eight months later, the fourth episode of aseptic meningitis occurred, which was negative for virus etiology (Table 1). She presented with a severe, throbbing headache, fever, and positive Kernig's and Brudzinski sign. Her symptoms improved with supportive treatment within three days. She continues to manage the intractable migraines with analgesics and Botox.

\section{Discussion}

Mollaret's meningitis is known for being recurrent but benign, with no long-term complications. Diagnosis is typically made after three episodes of aseptic meningitis with the characteristic pattern of the total 
resolution of symptoms between episodes. Mollaret's can present with transient neurological deficits but no prolonged or long-term residual complication. Our patient developed chronic intractable migraine with aura after the first episode of aseptic meningitis. Her migraine got worse with each episode of meningitis, causing dependence on multiple medications, psychological stress in the setting of constant pain, and multiple hospitalizations.

In 1981, Bruce H. Dobkin discussed that aseptic meningitis can produce a secondary migraine by causing the vasomotor instability of meningeal circulation by the inflammation of small, penetrating meningeal vessels to the cortex [2]. This mechanism of developing a migraine could be applied to this case. Our patient also developed papilledema, which is defined as edema of the optic disc as the result of elevated intracranial pressure (ICP). Elevated ICP can present without papilledema; however, papilledema cannot be present without elevated ICP as per the definition of papilledema [3-4]. However, papilledema is extremely rare and transient in aseptic meningitis and most of these cases have normal ICP. The mechanism of development of papilledema in aseptic meningitis is likely due to pleocytosis and meningeal inflammation rather than elevated ICP [4]. ICP can transiently increase in acute meningitis, which eventually normalizes. Our patient has developed papilledema with normal ICP after the third episode as the result of recurrent aseptic meningitis. However, subsequent episodes were not investigated for papilledema. In this very rare presentation of Mollaret's meningitis, chronic meningeal inflammation may have caused papilledema and migraine. Our patient also developed myoclonic jerk, ptosis, and tinnitus.

Recurrent episodes of meningitis can lead to intractable migraine with increasing severity with each episode and can produce resistance to analgesics. Over time, the patient might require a higher dose of opioids. Topiramate and Botox injections helped with migraine prophylaxis and treatment by decreasing the need for a higher dose of opioids and other analgesics. In our case, Botox was proven more beneficial for controlling symptoms.

\section{Etiology}

This patient had aseptic meningitis five times in a five-year period, three of which were associated with HSV-2. This is consistent with HSV-2 being the most commonly isolated pathogen in Mollaret's meningitis (up to $85 \%$ of cases) [5]. Mollaret's meningitis is more common in females than in males [5]. Various etiologies have been associated with Mollaret's meningitis, including spinal injections, minor head trauma, postpartum pituitary necrosis, low serum immunoglobulin G (IgG), family association, and familial Mediterranean fever [1,6-10].

While HSV-2 is considered the major underlying etiology of Mollaret syndrome, patients do not necessarily have concurrent genital lesions, though some patients always present with genital ulcers before the onset of CNS symptoms [11].

\section{Diagnosis and pathophysiology}

Clinical presentation is similar to bacterial meningitis with headache, fever, and positive meningeal signs. A patient can have a transient neurological disturbance, such as seizures, hallucinations, diplopia, cranial nerve paresis, abnormal reflexes, or coma, but no permanent neurological sequel $[1,5,9]$. Characteristic features and diagnostic criteria (by Bruyn et al. in 1962) of Mollaret's meningitis are described in Tables 2-3.

1 Recurrent episodes of aseptic meningitis

2 Symptoms free interval between the episodes

3 Spontaneous remission of symptoms

4 Transient neurological symptoms in $50 \%$ of the cases

$5 \quad$ No permanent neurological sequelae

6 The probable causative agent is Herpes Simplex Type 2 virus

$7 \quad$ Fever (not always present)

8 Genital symptoms absent in at least half of the cases

9 There may be increased gamma globulin fraction in the CSF

\section{TABLE 2: Characteristic features of Mollaret's meningitis}

Note: Data from Galdi, Farazmand, Woolley, Kinghorn, Abou-Foul, Buhary, Gayed SL [4-5,11] 


\section{Cureus}

1 Recurrent episodes of severe headaches, meningismus, and fever.

2 CSF pleocytosis of lymphocytes, neutrophils, and endothelial cells

3 Symptom-free resolution of symptoms and signs during the episodes

4 Spontaneous resolution of symptoms and signs during the episodes

$5 \quad$ Absence of detectable etiology

\section{TABLE 3: Diagnostic criteria for Mollaret's meningitis}

Note: Piskin IE, Yarimay G. Mollaret meningitis [2]

Leukocytosis is usually present. Leukopenia and eosinophilia have been noted previously [1]. Imaging usually remains negative $[1,12]$. CSF analysis can show clear CSF, pleocytosis, lymphocytosis, unusual large lymphocytes or mononuclear cells, and endothelial cells (Mollaret cells) [1]. Elevated neutrophils in CSF have been noted in some cases [12]. There will be elevated protein >100 and normal glucose. CSF gamma globulin, IgG level, and oligoclonal IgG bands have been elevated in past cases $[1,4,8,13]$. Culture usually remains negative.

Mollaret cells are described as large monocytic cells with nuclei shaped like a bean, footprint, or cloverleaf, and deep nuclear clefts [14-15]. Large, degenerated monocytes that appear as "ghost cells" can be present as well [14]. These cells are highly fragile and difficult to see after 24 hours from the onset of the attack and require a special stain (Papanicolaou). Mollaret cells are formed when mononuclear cells have been invaded by a virus and have been seen in varicella-zoster virus meningitis, Sjogren's disease, neuro-Behcet disease, sarcoidosis, and Vogt-Koyanagi-Harada disease [4]. CSF analysis has shown a rise in the IgG index and constant oligoclonal bands in some cases $[1,13]$.

\section{Treatment}

Mollaret's meningitis is a self-limiting and benign disease and should be treated with supportive measures. It is often treated with seven to 14 days of IV acyclovir. Treatment with acyclovir or valacyclovir is controversial. A randomized control trial of valacyclovir as suppressive therapy for recurrent aseptic meningitis showed no prevention of recurrent meningitis, and it was not recommended in the treatment of HSV meningitis [16]. There has been debate on the utility of prophylactic oral acyclovir, famciclovir, or valacyclovir for patients with this condition. Some case reports have demonstrated that valacyclovir has provided long-term relief, though other studies have not indicated a significant impact from prophylaxis $[11,16]$. Colchicine has generally failed as prophylaxis, but some cases had the benefit of meningitis-free years $[10,12,17]$. One case of recurrent aseptic lymphocytic meningitis has been documented in the setting of familial Mediterranean fever and responded to prophylactic colchicine treatment [7]. One case report of recurrent HSV-2 genital ulcers is correlated with recurrent meningitis episodes in which chronic suppressive treatment with acyclovir $800 \mathrm{mg}$ daily showed significant improvement [11].

\section{Prevalence of conditions}

Table 4 lists the various conditions associated with Mollaret's meningitis based on our literature search. 


\section{Cureus}

1 HSV-2 (most common)

2 HSV-1

3 Varicella-Zoster virus

4 HSV-6

5 Dermoid or epidermoid cyst

6 HSV-2 genital ulcers

7 Enterovirus infection

8 Migraine syndrome

9 Familial Mediterranean fever

10 Intracranial or intraspinal cyst

11 EBV infection

12 Behcet's disease

13 Sarcoidosis

14 Systemic lupus erythematosus

15 Intracranial tumor

16 Drug-induced (penicillin, cephalosporin trimethoprim-sulfamethoxazole, NSAIDs, immune globin intravenous, OKT3)

17 Vogt-Koyanagi-Harada syndrome

18 Whipple disease

19 Allergic disease

20 Postpartum pituitary necrosis

\section{TABLE 4: Conditions associated with Mollaret's meningitis}

HSV (herpes simplex virus), EBV (Epstein-Barr virus), NSAIDs (non-steroidal anti-inflammatory drugs), OKT (Orthoclone OKT3\{muromonab-CD3\}) Note: Data from $[1-2,4,7-12,14-15,18-20]$

\section{Conclusions}

It is important to diagnose this syndrome early to reduce tests and antibiotic use, but this rare meningitis with subsequent migraine makes the diagnosis challenging. Mollaret's is a benign syndrome with a good prognosis, but our case is unusual with severe migraine as a complication. Important considerations include a dilated fundus exam to look for papilledema and a Botox injection as treatment for chronic intractable migraine. Post-Mollaret's migraine should be differentiated from another attack of Mollaret's meningitis and symptoms should be treated adequately.

\section{Additional Information}

\section{Disclosures}

Human subjects: Consent was obtained by all participants in this study. Conflicts of interest: In compliance with the ICMJE uniform disclosure form, all authors declare the following: Payment/services info: All authors have declared that no financial support was received from any organization for the submitted work. Financial relationships: All authors have declared that they have no financial relationships at present or within the previous three years with any organizations that might have an interest in the submitted work. Other relationships: All authors have declared that there are no other relationships or activities that could appear to have influenced the submitted work.

\section{Acknowledgements}

Thanks to Ms. Laurie Schwing M.L.S. (Manager, Library Services) at UPMC Pinnacle, Harrisburg, PA, USA. 


\section{References}

1. Tyler KL, Adler D: Twenty-eight years of benign recurring Mollaret meningitis . Arch Neurol. 1983, 40:42-43. 10.1001/archneur.1983.04050010062017

2. Dobkin BH: Migraine and meningitis. Arch Neuro. 1981, 38:69. 10.1001/archneur.1981.00510010095032

3. Sengupta S, Eckstein C, Collins T: The dilemma of diagnosing idiopathic intracranial hypertension without papilledema in patients with chronic migraine. JAMA Neurol. 2019, 76:1001-1002. 10.1001/jamaneurol.2019.1696

4. Rigi M, Almarzouqi SJ, Morgan ML, Lee A: Papilledema: epidemiology, etiology, and clinical management . Eye Brain. 2015, 17:47-57. 10.2147/EB.S69174

5. Farazmand P, Woolley PD, Kinghorn GR: Mollaret's meningitis and herpes simplex virus type 2 infections . Int J STD AIDS. 2011, 22:306-307. 10.1258/ijsa.2010.010405

6. Wright WF, Palisoc K, Baghli S: Mollaret meningitis. J Neurol Sci. 2019, 15:148-149. 10.1016/j.jns.2018.11.018

7. Vilaseca J, Tor J, Guardia J, Bacardi R: Periodic meningitis and familial Mediterranean fever . Arch Intern Med. 1982, 142:378-379. 10.1001/archinte.1982.00340150178032

8. Haynes BF, Wright R, McCracken JP: Mollaret meningitis: a report of three cases . JAMA. 1976, 236:19671969. 10.1001/jama.1976.03270180043019

9. Capouya JD, Berman DM, Dumois JA: Mollaret's meningitis due to human herpes virus 6 in an adolescent . Clin Pediatr. 2006, 45:861-863. 10.1177\%2F0009922806295286

10. Barakat MH, Mustafa HT, Shakir RA: Mollaret's meningitis. A variant of recurrent hereditary polyserositis, both provoked by metaraminol. Arch Neurol. 1988, 45:926-927. 10.1001/archneur.1988.00520320128030

11. Abou-Foul AK, Buhary TM, Gayed SL: Herpes simplex virus type 2-associated recurrent aseptic (Mollaret's) meningitis in genitourinary medicine clinic: a case report. Int Med Case Rep J. 2014, 7:31-33. 10.2147\%2FIMCRJ.S58377

12. Piskin IE, Yarimay G: Mollaret meningitis: a case report. Turk J Pediatr. 2010, 52:306-308.

13. Barontini F, Ghezzi M, Marconi GP: A case of benign recurrent meningitis of Mollaret . J Neurol. 1981, 225:197-206. 10.1007/BF00313749

14. Jones CW, Snyder GE: Mollaret meningitis: case report with a familial association. Am J Emerg Med. 2011, 29:840-841. 10.1016/i.ajem.2010.02.008

15. Livingston RA, Harrison CJ: Recurrent meningitis. Principles and Practice of Pediatric Infectious Disease, 5th ed. Long SS, Prober CG, Fischer M (ed): Elsevier, Inc., Philadelphia; 2018. 295-300.

16. Aurelius E, Franzen-Rohl E, Glimaker M, et al.: Long-term valacyclovir suppressive treatment after herpes simplex virus type 2 meningitis: a double-blind, randomized controlled trial. Clin Infect Dis. 2012, 54:13041313. 10.1093/cid/cis031

17. Stamm AM, Livingston WK, Cobb CG, Dismukes WE: Failure of colchicine in the treatment of Mollaret's meningitis. Arch Intern Med. 1984, 144:2265-2266. 10.1001/archinte.1984.04400020194033

18. Ohmichi T, Takezawa H, Fujii C, Tomii Y, Yoshida T, Nakagawa M: Mollaret cells detected in a patient with varicella-zoster virus meningitis. Clin Neurol Neurosurg. 2012, 114:1086-1087. 10.1016\%2Fj.clineuro.2012.02.015

19. Mikdashi J, Kennedy S, Krumholz A: Recurrent benign lymphocytic meningitis in systemic lupus erythematosus. Neurologist. 2008, 14:43-45. 10.1097/NRL.0b013e318157a669

20. Graman PS: Mollaret's meningitis associated with acute Epstein-Barr virus mononucleosis . Arch Neurol 1987, 44:1204-1205. 10.1001/archneur.1987.00520230084023 